\title{
Atmospheric Pressure Photoionization as a Powerful Tool for Large-Scale Lipidomic Studies
}

\author{
Mathieu Gaudin, ${ }^{1,2,3}$ Laurent Imbert, ${ }^{4}$ Danielle Libong, ${ }^{4}$ Pierre Chaminade, ${ }^{4}$ \\ Alain Brunelle, ${ }^{1}$ David Touboul, ${ }^{1}$ Olivier Laprévote ${ }^{2}$ \\ ${ }^{1}$ Centre de Recherche de Gif, Institut de Chimie des Substances Naturelles, CNRS, Gif-sur-Yvette, France \\ ${ }^{2}$ Chimie-Toxicologie Analytique et Cellulaire, EA 4463, Faculté des Sciences Pharmaceutiques et Biologiques, \\ Université Paris Descartes, Sorbonne Paris Cité, Paris, France \\ ${ }^{3}$ Division Métabolisme, Technologie Servier, Orléans, France \\ ${ }^{4}$ Groupe de Chimie Analytique de Paris Sud, EA 4041, Faculté de Pharmacie, Université Paris-Sud, Châtenay-Malabry, France
}

\begin{abstract}
Lipidomic studies often use liquid chromatography/electrospray ionization mass spectrometry (LC/ESI-MS) for separation, identification, and quantification. However, due to the wide structural diversity of lipids, the most apolar part of the lipidome is often detected with low sensitivity in ESI. Atmospheric pressure (APPI) can be an alternative ionization source since normal-phase solvents are known to enhance photoionization of these classes. In this paper, we intend to show the efficiency of APPI to identify different lipid classes, with a special interest on sphingolipids. In-source APPI fragmentation appears to be an added value for the structural analysis of lipids. It provides a detailed characterization of both the polar head and the non polar moiety of most lipid classes, and it makes possible the detection of all lipids in both polarities, which is not always possible with ESI.
\end{abstract}

Key words: Lipid, APPI, In-source fragmentation

\section{Introduction}

T ipids, which can be defined as biological, hydrophoLic, or amphiphilic substances mainly soluble in organic solvents [1] are of great interest since they are involved in various biological functions. They are major constituents of cell membrane bilayer of which the fluidity is determined by its lipid composition [2]. The distribution of specific lipid species, such as cholesterol

Electronic supplementary material The online version of this article (doi:10.1007/s13361-012-0341-y) contains supplementary material, which is available to authorized users.

Correspondence to: David Touboul; e-mail: touboul@icsn.cnrs-gif.fr and sphingolipids, is responsible for the formation of organized membrane microdomains called rafts [3]. Lipids also provide a hydrophobic environment necessary to membrane protein function and interact with hydrophobic residues. They also constitute an efficient energy source [4] and a transporter of dietary fat. Finally, they are involved in signaling pathways as a source of secondary messengers. For example, eicosanoids, such as prostaglandins, are metabolites of free fatty acids and have a potent pro-inflammatory activity [5]. A better understanding of lipid biology consequently requires analytical methods to qualitatively and quantitatively determine lipid composition.

Due to the complexity of biological lipid extracts, numerous separation techniques have been used such as 
thin layer chromatography (TLC) [6], gas chromatography (GC) [7], normal-phase liquid chromatography (NPLC) [8], or reverse-phase liquid chromatography (RPLC) [9]. In order to detect and elucidate the structure of minor lipid species, mass spectrometry has been largely used, and liquid chromatography/mass spectrometry has become the gold standard for lipidomic analysis thanks to its sensitivity and specificity.

Electrospray (ESI) [10] or atmospheric pressure chemical ionization (APCI) [11] can be considered as efficient ionization techniques to study phospholipids and sphingolipids but the analysis of apolar compounds, such as triacylglycerols, remains challenging without addition of cationizing agents (ammonium [12], lithium [13], or silver [14]). On the contrary, atmospheric pressure photoionization (APPI) [15] is of growing interest for analyzing apolar compounds. Studies of highly hydrophobic compounds, such as halogenated flame retardants [16] and polycyclic aromatic hydrocarbons [17] have revealed the excellent suitability of APPI for the analysis of persistent organic pollutants. Nevertheless, more polar compounds soluble in water/methanol mixture (i.e., steroids [18], peptides [19, 20], quaternary ammonium salts [21], oligosaccharides [22], or oligodeoxyribonucleotides [23]) are also ionizable with APPI. This feature enables the efficient coupling of both reverse-phase and normal-phase liquid chromatography with APPI-MS [24]. These two chromatographic modes are frequently used to analyze lipids. However, as far as we know, only few different lipid classes have already been studied by APPI-MS (i.e., acylglycerols [25], sterols [26], glycosphingolipids [27, 28]. or phospholipids [29].

In this study, we demonstrate how efficient APPI can be for the identification of a wide range of different lipids, from apolar triterpenes up to the more polar sphingomyelins, thus providing an improvement in sensitivity for the detection of non-polar classes in comparison with ESI.

Moreover, in-source fragmentations have been systematically examined leading to the conclusion that lipids display different behaviors under APPI conditions compared to ESI ones. Unusual mechanisms, such as electron capture dissociation [19] and homolytic bond cleavages, will be especially highlighted.

\section{Experimental}

\section{Chemicals and Sample Preparation}

Acetone, n-heptane, ethyl acetate, and isopropanol (HPLC grade) were purchased from Carlo Erba (Val de Reuil, France). Water was obtained from a MilliQ Apparatus (Millipore, Saint-Quentin en Yvelines, France). Acetic acid (LC/MS grade) was from Fluka (Saint-Quentin Fallavier, France). Phospholipid and sphingolipid standards were purchased from Avanti Polar Lipids (Alabaster, AL, USA). Triacylglycerol, cholesterol, stearic acid, and squalene were from Sigma-Aldrich (Saint-Quentin Fallavier, France). Stock solutions of lipid standards were either prepared in pure chloroform or in a chloroform/methanol/water mixture (50/ $40 / 10, \mathrm{vol} / \mathrm{vol} / \mathrm{vol}$ ) at a concentration of $2 \mathrm{mM}$. Further dilutions were done in isopropanol.

\section{Liquid Chromatography}

Normal-phase liquid chromatography was performed on an Agilent 1100 series HPLC system (Agilent, Palo Alto, CA, USA), according to a method adapted from Greave et al. [30] Briefly, two Chromolith Performance Si columns (Merck, Darmstadt, Germany), $100 \times 4.6 \mathrm{~mm}$ are set-up in series at fixed temperature of $40{ }^{\circ} \mathrm{C}$. Eluent flow rate was $1 \mathrm{~mL} \cdot \mathrm{min}^{-1}$. Ternary gradient elution was performed using mixtures of A: heptane/ethyl acetate 99.8/0.2; B: acetone/ ethyl acetate 2/1; C: isopropanol/water $85 / 15$. Acetic acid $0.05 \%$ was added to both eluents B and C. Injection volume was $5 \mu \mathrm{L}$. Acetone acting as a dopant was post-column infused at a flow rate of $50 \mu \mathrm{L} \cdot \mathrm{min}^{-1}$.

\section{Mass Spectrometry}

Ionization was achieved with a PhotoMate krypton lamp (Syagen Technologies, Tustin, CA, USA) mainly emitting $10.0 \mathrm{eV}$ photons with a minor contribution at $10.6 \mathrm{eV}$. Mass spectra were acquired on a triple quadrupole mass spectrometer (TSQ Vantage EMR; Thermo Fisher, San Jose, CA, USA) in full scan mode. Peak width was set at $0.7 \mathrm{Da}$. For LC/MS profiling experiments, due to high noise level below $m / z 200$, the $\mathrm{m} / \mathrm{z}$ range was fixed at $300-1500$ in positive ion mode and at $200-1500$ in negative ion mode. For detailed study of in-source fragmentation, the $\mathrm{m} / \mathrm{z}$ range was extended to $50-1500$ in both ion polarities. Vaporizer and capillary temperature were set at 325 and $350{ }^{\circ} \mathrm{C}$, respectively. Sheath and auxiliary gas were fixed at 50 and 30 arbitrary units, respectively. S-lens voltage was set at $145 \mathrm{~V}$. High resolution mass spectra of phosphatidylcholines and sphingomyelins were also acquired in negative ion mode with a LTQ-Orbitrap mass spectrometer (Thermo Fisher, San Jose, CA, USA), using the same Syagen APPI device. Collision-induced dissociation was achieved with helium as collision gas. The collision energy was set at 19 arbitrary units and the activation time at $30 \mathrm{~ms}$.

\section{Results and Discussion}

An equimolar mixture of lipid standards has been analyzed by normal-phase liquid chromatography-atmospheric pressure photoionization-mass spectrometry, both in positive and negative ion modes (Figure S-1). A higher sensitivity is observed for compounds from medium polar classes (e.g., ceramides) than for polar compounds (e.g., phosphatidylcholines, sphingomyelins). This can be explained by the high proportion of acetone in mobile 
phase at these retention times, acting as a dopant enhancing the ionization efficiency.

\section{Positive Ion Mode}

\section{Triterpenes}

In our study, this lipid class is illustrated by squalene, an early precursor in the cholesterol biosynthesis pathway, also used in vaccines as an adjuvant [31]. This molecule is formed by the condensation of isopentenyl pyrophosphate and dimethylallyl pyrophosphate moieties [32]. Squalene has already been analyzed by gas chromatography/mass spectrometry (GC/MS) with electron impact (EI) ionization [33], leading to the detection of the $\mathrm{M}^{+}$ion at $m / z 410$, or by HPLC with UV detection [34]. The absence of acidic or basic sites makes this molecule poorly ionizable by ESI. Under APPI conditions, the positive-ion mass spectrum exhibits an intense ion peak signal at $\mathrm{m} / \mathrm{z} 411.4$ corresponding to the protonated molecule $[\mathrm{M}+\mathrm{H}]^{+}$. An ion peak at $\mathrm{m} / \mathrm{z} 410.4$ could have been expected by direct photoionization leading to the formation of $\mathrm{M}^{+\cdot}$ since the ionization potential of 2-methylpent-2-ene, the monomer moiety of squalene, is $8.58 \mathrm{eV}$, (i.e., significantly lower than the energy of the photons emitted by the krypton lamp. As an $[\mathrm{M}+\mathrm{H}]^{+}$ion is detected, a classic two stage process [35] can be considered, combining a direct photoionization of the molecule $M$ (equation 1) and a proton transfer from the solvent $\mathrm{S}$ (equation 2):

$$
\begin{gathered}
M+h v \rightarrow M^{\bullet+}+e^{-} \\
M^{\bullet+}+S \rightarrow[M+H]^{+}+[S-H]^{\bullet}
\end{gathered}
$$

However, in the case of our solvent system, reaction 2 is endothermic $\left(\Delta \mathrm{H}=82.7 \mathrm{~kJ}^{\mathrm{mol}}{ }^{-1}\right)$, eliminating this hypothesis. We thus propose that protonated squalene arises from a proton transfer after photoionization of heptane according to the following reactions:

$$
\begin{gathered}
S+h v \rightarrow S^{\bullet+}+e^{-} \\
S^{\bullet+}+S \rightarrow[S+H]^{+}+[S-H]^{\bullet} \\
M+[S+H]^{+} \rightarrow[M+H]^{+}+S
\end{gathered}
$$

Heptane exhibits an ionization potential of $9.93 \mathrm{eV}$ and can thus be photoionized by photons from a $\mathrm{Kr}$ lamp (equation 3). Ionized heptane would then react on neutral heptane to produce protonated heptane molecules (equation 4). Due to the lack of thermodynamic data on heptane, proton affinity of isobutane was used in the calculation of the reaction enthalpy $[36,37]$, leading to the conclusion that this reaction is exothermic $\left(\Delta \mathrm{H}=-228.8 \mathrm{~kJ} \cdot \mathrm{mol}^{-1}\right)$. Due to the fact that the proton affinity of 2-methylpent-2-ene $\left(812 \mathrm{~kJ}^{\left.-\mathrm{mol}^{-1}\right)}\right)$ is larger than the one of heptane $(677.8 \mathrm{~kJ}$. $\mathrm{mol}^{-1}$ for isobutane), a proton transfer can occur, leading to the formation of $[\mathrm{M}+\mathrm{H}]^{+}$ion (equation 5). Thus atmospheric pressure photoionization can be an efficient way to interface liquid chromatography to mass spectrometry for analysis of terpenes deprived of heteroatoms.

\section{Triacylglycerols}

Triacylglycerols (TG) are formed by esterification of three molecules of free fatty acids on a glycerol molecule. As mentioned above, their analysis is often difficult in ESI without addition of salts [25]. Moreover, the determination of the acyl chain lengths requires tandem mass spectrometry. By contrast, the APPI-MS spectrum of a TG compound $(\mathrm{C} 14: 0 / \mathrm{C} 14: 0 / \mathrm{C} 14: 0)$ displays a weak $[\mathrm{M}+\mathrm{H}]^{+}$ion peak signal at $m / z$ 723.6. This behavior has been shown to be typical of saturated triacylglycerols [38]. A major fragment ion signal is observed at $\mathrm{m} / \mathrm{z} 495.3$ arising from the loss of one of the acyl chains as a carboxylic acid C14:0. The second fragment ion signal at $\mathrm{m} / \mathrm{z} 285.2$ reveals the loss of a second fatty acid moiety as a ketene. Thus, the three fatty acid chain lengths can be determined by APPI-MS according to in-source fragmentation. Regioisomers can also be distinguished since fatty acid neutral losses from $s n-1$ or $s n-3$ position are favored over the $s n-2$ position [38]. APPI-MS can thus be an interesting tool to elucidate the structure of an unknown TG without using tandem mass spectrometry.

\section{Sterols and Oxysterols}

Cholesterol and other members of the sterol family play an important role in membrane structure and fluidity and are involved in various diseases such as atherosclerosis [39] and Alzheimer's disease [40]. Their analysis is complicated by poor ionization yields in ESI and by the structural diversity due to numerous isomers. Under APPI conditions and in positive ion mode, the major ion arises from a dehydration reaction of the protonated molecule $\left(\left[\mathrm{M}+\mathrm{H}-\mathrm{H}_{2} \mathrm{O}\right]^{+}\right)$. These kinds of dehydrated ions are identical to those seen in APCI [26], but with a 10 -fold increase in signal-to-noise ratio.

No $[\mathrm{M}+\mathrm{H}]^{+}$ion are detected but some minor ion peaks are also present in the spectrum. The ion signal at $\mathrm{m} / \mathrm{z} 383.3$ corresponds formally to $\left[\mathrm{M}+\mathrm{H}-2 \mathrm{H}_{2}\right]^{+}$, which is assumed to be a mono-unsaturated five-membered ring derivative of cholesterol. Further desaturation is observed leading to a conjugated di-unsaturated ring as observed by Varga et al. [41] on the side chain of ergosterol under positive ion APPI conditions. 


\section{Phosphatidylcholine}

Phosphatidylcholines (PC) are the major constituents of the plasma membrane of mammalian cells. Due to their quaternary ammonium, these lipids are easily detected in the positive ion mode. We previously demonstrated by flow injection analysis the potential of APPI for the structural elucidation of PC [29]. The main observed feature (Figure S-2) was the in-source neutral loss of phosphocholine (-183 Da) in APPI instead of the formation of the protonated phosphocholine at $\mathrm{m} / z 184$ in ESI-MS/MS [42, 43]. The fragmentation behavior in APPI is similar to those observed in CID experiments for sodium and lithium cationized PC obtained by ESI. Besides, a regioselective fragmentation makes unambiguous assignment of acyl chain positions possible. After coupling with normal-phase liquid chromatography method, similar results were obtained allowing APPI to analyze PC in a biological mixture. A specific fragmentation pathway described for APPI-MS of phosphatidylcholines is a demethylation of both the protonated and sodium-cationized species producing $N$-dimethyl-phosphatidylethanolamine. The polar head group is detected thanks to the ion peaks at $m / z 60.1$ and 72.1 , corresponding to protonated trimethylamine and protonated $\mathrm{N}$-dimethyl-ethenamine, respectively. In the low mass range of ESI-MS/MS spectrum of protonated PC, ion peaks at $m / z 86$ and 125 are observed, corresponding to fragments of the polar head group retaining the quaternary ammonium or the phosphate group, respectively [42]. The ion at $m / z 72$ arises from demethylated PC and is exclusively specific to APPI-MS analysis. All other peaks are shared with other phospholipid classes and will be discussed below.

\section{Other Phospholipids}

Phosphatidylethanolamines (PE), phosphatidylserines (PS), phosphatidylglycerols (PG), phosphatidylinositols (PI), and phosphatidic acids (PA) have also been investigated. Protonated or sodium cationized molecules of PE and PG have been weakly detected but in-source fragmentations remain important for these families under APPI conditions (Figure 1). Although PG [44], PI [45], and PA [46] are traditionally analyzed in the electrospray negative ion mode, due to their anionic polar head group, these photoionized species have been successfully detected in positive ion mode. Because of in-source dissociation, positively charged fragments of these acidic species are formed, allowing the detection of all the phospholipid families with a single polarity. For all phospholipids, the base peak corresponds to the ion formed by the loss of the polar head group from the protonated molecule. PE, PS, PG, PI, and PA lose phosphoethanolamine $\left(\mathrm{C}_{2} \mathrm{H}_{8} \mathrm{NO}_{4} \mathrm{P},-141 \mathrm{Da}\right)$, phosphoserine $\left(\mathrm{C}_{3} \mathrm{H}_{8} \mathrm{NO}_{6} \mathrm{P},-185 \mathrm{Da}\right)$, phosphoglycerol $\left(\mathrm{C}_{3} \mathrm{H}_{9} \mathrm{O}_{6} \mathrm{P},-\right.$ $172 \mathrm{Da})$, phosphoinositol $\left(\mathrm{C}_{6} \mathrm{H}_{13} \mathrm{O}_{9} \mathrm{P},-260 \mathrm{Da}\right)$, and phosphoric acid $\left(\mathrm{H}_{3} \mathrm{PO}_{4},-98 \mathrm{Da}\right)$, respectively [47].

This major $[\mathrm{M}+\mathrm{H}-\text { head group }]^{+}$ion, which retains the glycerol backbone, undergoes further dissociation leading to minor fragments arising from the loss of the fatty acyl substituents as ketenes. Contrary to ESI-MS/MS of phospholipids [10], neutral fatty acid loss is not observed. For the 16:0/18:1 phospholipids (Figure 1a-c) ions peaks appear at $\mathrm{m} / \mathrm{z} 313.2$ and 339.2 arising, respectively, from the loss of C18:1 and C16:0 ketenes. For the PI(18:1/18:1) (Figure 1d), a single ion peak at $m / z 339.2$ is observed since acyl chains at $s n-1$ and $s n-2$ positions are the same. The acyl chain lengths of phospholipids can thus be determined easily but the lack of specificity of these fragmentations does not allow the assignment of their position on the glycerol backbone. As intact species are not always present in the spectrum and polar head group-related fragments are not observed, even in the low mass range, unambiguous identification of the head group requires a comparison of the retention times in normal-phase liquid chromatography with an authentic standard of this family. These different phospholipid species are indeed efficiently separated under the chromatographic conditions reported in the Experimental section. Finally, in positive ion mode, sensitivity decreases as follows: PC> PE $>$ PG $>$ PS $>$ PA. This can be explained by the ability of these phospholipids to acquire a positive charge. $\mathrm{PC}$ is permanently charged due to its quaternary ammonium. Then sensitivity decreases with decreasing gas phase basicity for the other four phospholipid families.

\section{Ceramides}

Ceramides (Cer) constitute one of the simplest sphingolipids. They are composed of a sphingosine backbone, characterized by a secondary alcohol function and an amine linked to a fatty acid chain by an amide bond, contrary to the glycerolipids for which ester linkage is predominant. The ceramide moiety ends with a primary alcohol, which can be functionalized to give other sphingolipids classes such as sphingomyelins or glycosphingolipids. The APPI-MS spectrum of Cer (d18:1/24:1) displays minor ion signals at $\mathrm{m} / \mathrm{z}$ 648.6 and 670.6 , which are assigned to the protonated and sodium cationized molecules, respectively. The mass spectrum is dominated by a peak at $m / z 630.6$ corresponding to $\left[\mathrm{M}+\mathrm{H}-\mathrm{H}_{2} \mathrm{O}\right]^{+}$. A second dehydration step also occurs, leading to the ion signal at $\mathrm{m} / \mathrm{z}$ 612.6. This confirms the presence of two hydroxyl groups on the ceramide moiety. Dehydration is favored by the formation of a conjugated system on the ceramide backbone. This is in agreement with a previous work of Muñoz-Garcia et al. [48], who used PhotoSpray toluene-assisted APPI-MS to study the ceramide and cerebroside content of the stratum corneum. In APPIMS, dehydration seems to be frequent for hydroxyl-rich molecules, whatever the source design (Photomate or PhotoSpray). This phenomenon may be due to thermal dissociation processes [49]. Besides, we also detected an insource fragment ion peak at $m / z 264.3$, which appears to be a characteristic signature of the ceramide backbone since it can be attributed to a [Sphingosine $\left.+\mathrm{H}-2 \mathrm{H}_{2} \mathrm{O}\right]^{+}$. An 
(a)

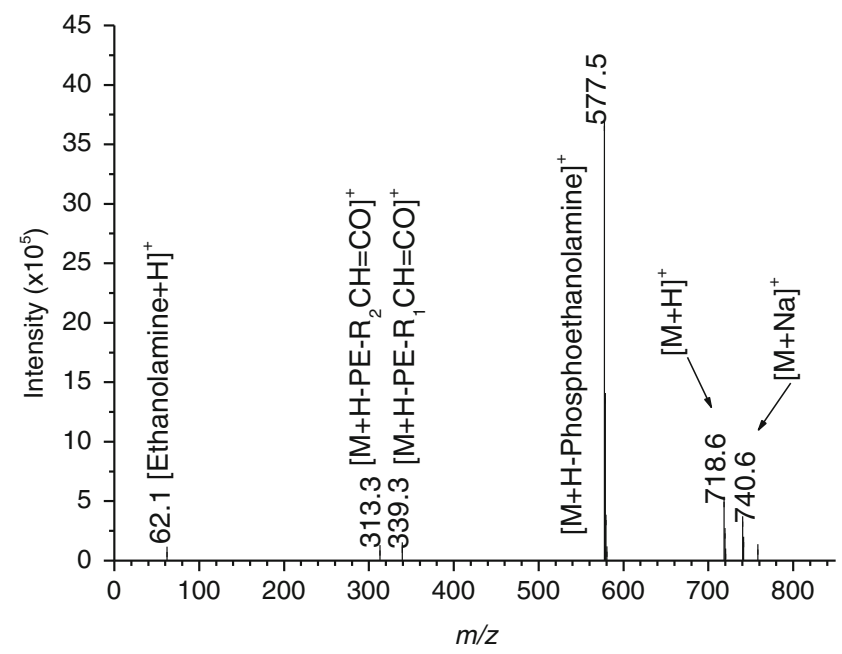

(c)

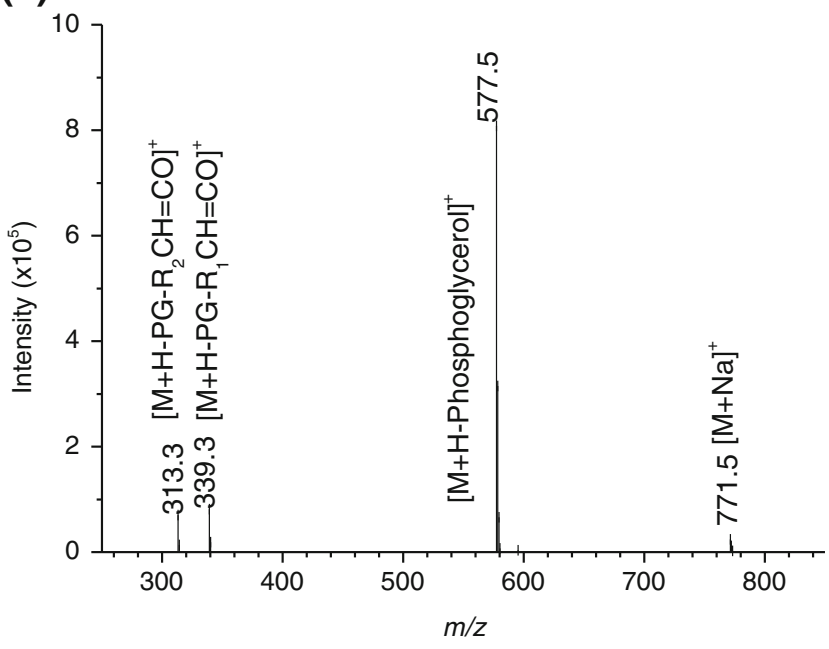

(b)

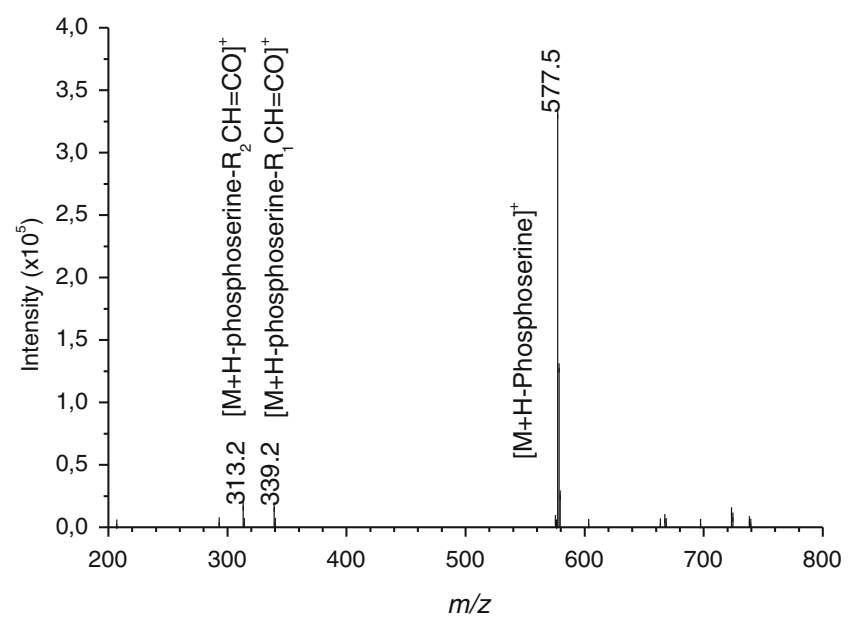

(d)

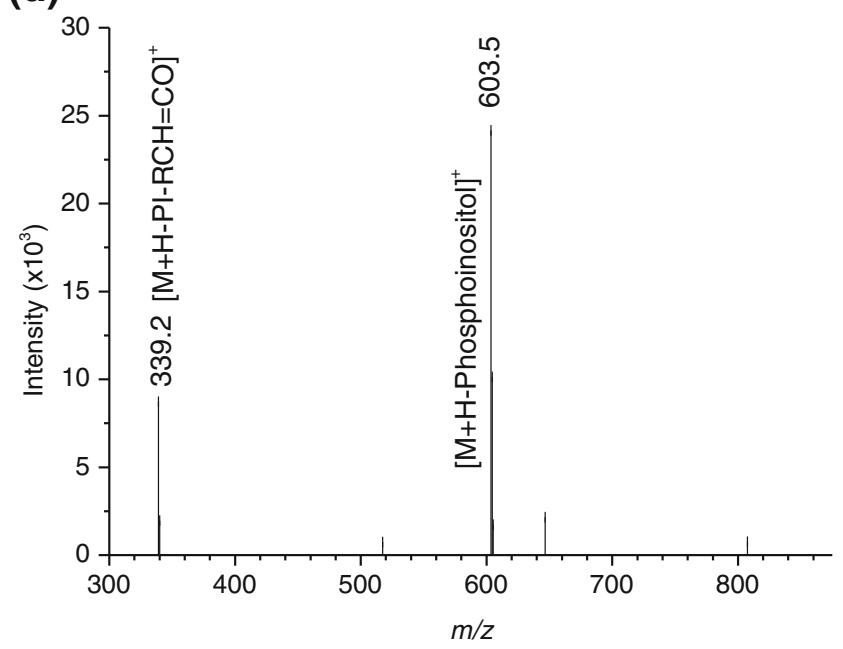

Figure 1. Positive-ion mode APPI mass spectra of: (a) phosphatidylethanolamine (16:0/18:1); (b) phosphatidylserine (16:0/ 18:1); (c) phosphatidylglycerol (16:0/18:1); (d) phosphatidylinositol (18:1/18:1). All phospholipid APPI spectra are dominated by an ion arising from the neutral loss of the polar head group. Further losses of ketenes from this ion allow the determination of fatty acid composition

aziridine structure has been proposed by Hsu et al. [50] for this ion when obtained by ESI-MS/MS. This ion can be used to mine the LC-APPI-MS data in order to find all d18:1 sphingolipids without running a precursor ion scan experiment with a triple quadrupole mass spectrometer.

\section{Galactocerebrosides}

Galactocerebrosides or galactosylceramides (GalCer) are glycosphingolipids formed by a glycosidic linkage between galactose and the primary hydroxyl of a ceramide. Consequently, they are more polar than ceramides leading to longer retention time on normal phase liquid chromatography. The intact GalCer (d18:1/24:1) ion is detected as a sodium adduct at $\mathrm{m} / \mathrm{z} 832.6$ (Figure 2), whereas no protonated species is detected. This behavior is typical of oligosaccharides which are known to have low basicity and high alkaline metal ion affinity [51]. Dehydration from sodium-cationized GalCer and from instable protonated GalCer leads respectively to the minor ion peak at $\mathrm{m} / \mathrm{z}$ 814.6 and to the base peak at $\mathrm{m} / z$ 792.6. A weak ion signal corresponding to $\left[\mathrm{M}+\mathrm{Alk}-\mathrm{H}_{2} \mathrm{O}\right]^{+}$has already been observed in ESI tandem mass spectrometric analysis of lithiated GalCer [52], but in that study, no $\left[\mathrm{M}+\mathrm{H}-\mathrm{H}_{2} \mathrm{O}\right]^{+}$ ion was present contrary to what is observed in APPI. A pair of ion peaks at $\mathrm{m} / \mathrm{z} 612.6$ and 630.6, arising from the galactose loss, identifies the ceramide dehydrated fragments as mentioned above [21]. Finally, as a member of the sphingolipid family, the GalCer APPI mass spectrum 


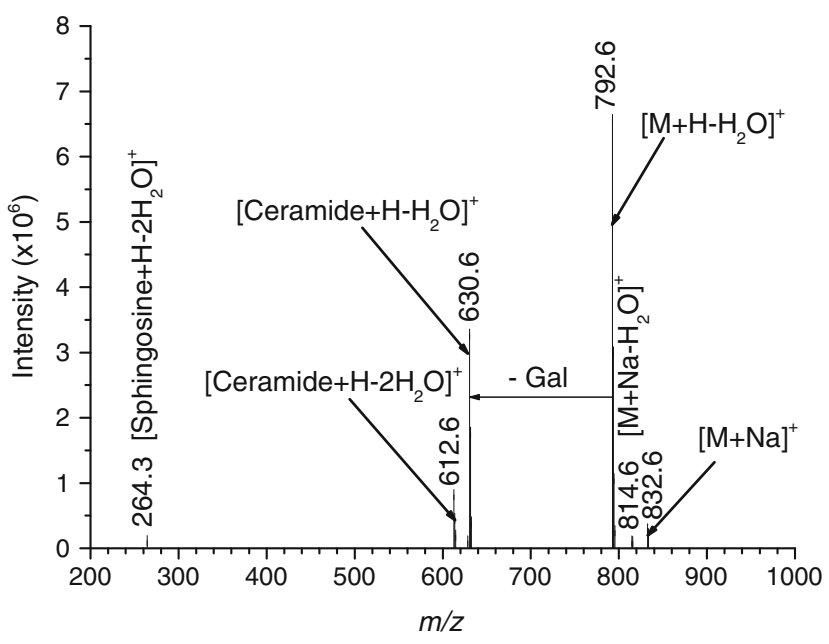

Figure 2. Positive-ion mode APPI mass spectrum of galactocerebroside (d18:1/24:1). The base peak corresponds to dehydrated GalCer, reflecting the thermal processes involved in atmospheric pressure photoionization. A neutral loss of galactose is observed leading to ceramide-related ions

exhibits the characteristic ion peak at $\mathrm{m} / \mathrm{z} 264.3$ corresponding to the di-dehydrated sphingosine ion.

\section{Sulfatides}

Sulfatides (ST) are sulfated galactosylceramides of major interest in several pathologies such as Alzheimer's disease [53]. They are usually analyzed in the negative ion mode due to their acidity. Their behavior under APPI conditions has never been described before. APPI-MS enables the sensitive detection of these lipids in both ion polarities due to in-source fragmentations. The positive ion mass spectra of sulfatides are similar to those of galactocerebrosides, confirming that the sulfate group is very labile, meaning that only retention times in normal phase liquid chromatography are able to differentiate these two families in the positive ion mode.

\section{Sphingomyelin}

Sphingomyelins are phosphocholine-containing sphingolipids. They thus exhibit both PC-like and sphingolipid-like fragmentation behavior (Figure 3). The intact SM (d18:1/ 24:1) ion is detected as a sodium cationized species at $\mathrm{m} / \mathrm{z}$ 835.6. The ion at $\mathrm{m} / \mathrm{z} 776.6$ is formed by the elimination of trimethylamine $\left([\mathrm{M}+\mathrm{Na}-59]^{+}\right)$. This neutral loss is also observed for phosphatidylcholines [15]. The major fragment ions at $\mathrm{m} / \mathrm{z} 612.6$ and 630.6 represent the ceramide moiety after loss from the protonated SM of the phosphocholine head group followed by one or two dehydrations, respectively, as previously described for Cer, GalCer, and ST. As they belong to the sphingolipid class, the photo-ionized sphingomyelins lead to the following ion series: the ion at $\mathrm{m} /$

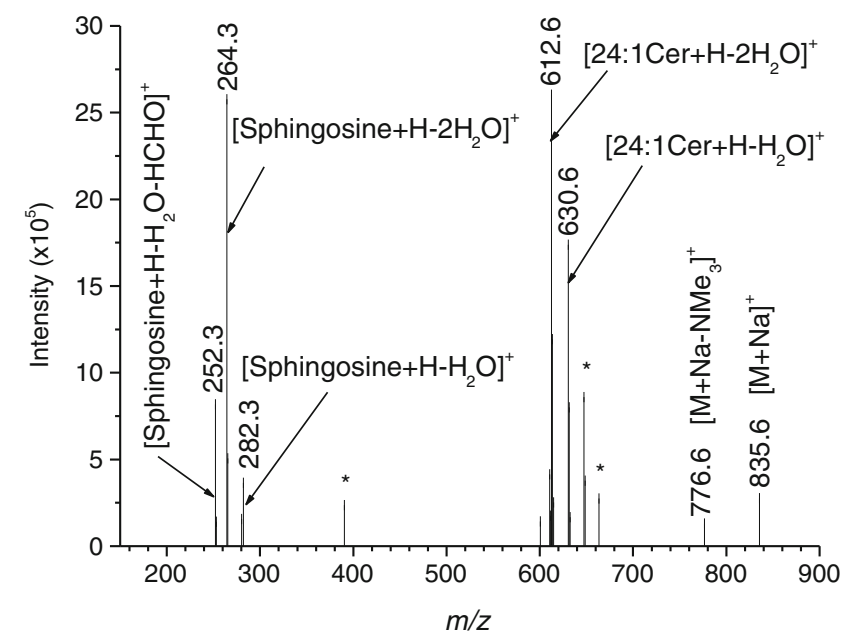

Figure 3. Positive-ion mode APPI mass spectrum of sphingomyelin (d18:1/24:1). Impurities are marked with an asterisk. The major fragmentation pathways involve loss of the phosphocholine head group and dissociation of the ceramide backbone leading to the diagnostic ion at $\mathrm{m} / \mathrm{z} 264.3$ that can be considered as a hallmark of the sphingolipids

$z 282.3$ corresponding to mono-dehydrated sphingosine ion can undergo either loss of water leading to the ion at $\mathrm{m} / \mathrm{z}$ 264.3, already present in the Cer and GalCer spectra, or loss of formaldehyde to form the ion at $\mathrm{m} / \mathrm{z}$ 252.3. These APPI-induced fragmentations differ from those obtained by CID after ESI, which are dominated by an ion signal at $\mathrm{m} / \mathrm{z} 184.1$ corresponding to the phosphocholine head group. No ion peak arising from the sphingosine backbone has been observed in positive ESI-MS/MS. As for PC, the fragmentation behavior in APPI is similar to those observed in CID experiments for lithiated SM obtained by ESI [54].

\section{Negative Ion Mode}

To date, few applications in lipidomics of negative atmospheric pressure photoionization have been reported. Previous studies were mainly focused on ceramides [48], cerebrosides [48], and globotriaosylceramides [27].

\section{Terpenes}

In the negative ion mode, APPI-MS analysis of squalene shows a single ion signal at $\mathrm{m} / \mathrm{z} 409.4$ corresponding to the deprotonated molecule, of which the formation could be explained by the following mechanism [55]. First, acetone, acting as dopant (D) is photoionized (equation 6) and produces low energy electrons. Due to its positive electron affinity (EA = $0.45 \mathrm{eV}$ ), molecular oxygen present in the atmospheric pressure source can capture these low energy electrons to produce a superoxide anion (equation 7). Finally, the analyte 
reacts with the superoxide anion leading to the hydroperoxy radical and deprotonated squalene (equation 8).

$$
\begin{gathered}
D+h v \rightarrow D^{\bullet+}+e^{-} \\
\mathrm{O}_{2}+e^{-} \rightarrow \mathrm{O}_{2}^{\bullet-} \\
M+\mathrm{O}_{2}^{\bullet-} \rightarrow[M-H]^{-}+\mathrm{HO}_{2}^{\bullet}
\end{gathered}
$$

\section{Phosphatidylcholines}

In the negative ion ESI mode, $\mathrm{PC}$ can only be ionized if an ion pairing agent $(\mathrm{RCOOH})$ such as formic acid or acetic acid is added [54]. Their respective carboxylate anions are able to hide the positively charged quaternary ammonium of the choline head group. PC is thus detected as a $[\mathrm{M}-\mathrm{H}+$ $\mathrm{RCOOH}]^{-}$ion after deprotonation of the phosphate group.

Contrary to ESI, the addition of acids is not necessary in negative ion mode APPI-MS to ionize PC, since the emission of low energy electrons enables by electron capture the formation of a $\mathrm{M}^{--}$molecular ion. With the low mass resolution of a triple quadrupole instrument, the ion peak at $\mathrm{m} / \mathrm{z} 759.5$ can be assigned either to the $\mathrm{M}^{--}$ion or to the acetic acid adduct of the ion at $\mathrm{m} / \mathrm{z} 699.5$ since mobile phases B and C contains $0.05 \%$ of acetic acid to enhance chromatographic performance (Figure 4).

Phosphatidylcholines in negative ion mode have thus been investigated by high resolution mass spectrometry with a LTQOrbitrap to resolve the ambiguity. The measured value of $\mathrm{m} / \mathrm{z}$ is

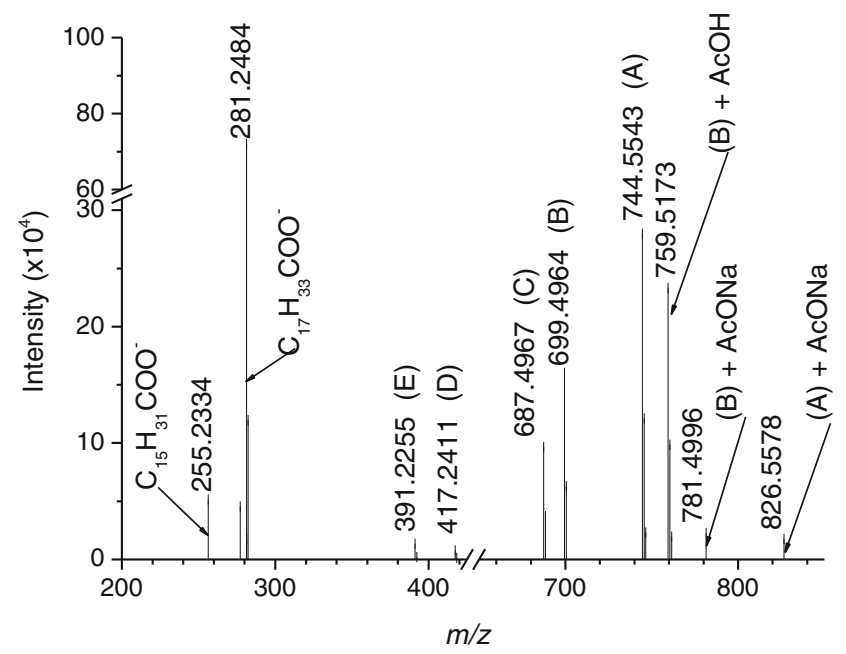

Figure 4. Negative-ion mode APPI mass spectrum of phosphatidylcholine (16:0/18:1). Major ions are carboxylate anions. Ion peaks $D$ and $E$ arise from fatty acid neutral losses allowing acyl chain length confirmation. Polar head group dissociations are also observed leading to ions $\mathrm{A}$ to $\mathrm{C}$ involving the losses of methyl radical or trimethylamine
759.5173. The deviation is $\Delta_{\mathrm{ppm}}=-80.4 \mathrm{ppm}$ from the calculated $\mathrm{m} / \mathrm{z}$ value of $\mathbf{M}^{--}$from the studied PC $(\mathrm{m} / \mathrm{z}$ 759.5784 ), whereas the deviation is only $\Delta_{\mathrm{ppm}}=0.3 \mathrm{ppm}$ with the calculated $\mathrm{m} / \mathrm{z}$ value (759.5173) of the acetic acid adduct of the ion at $m / z$ 699.5. However, if no ion peak corresponding to an instable $\mathrm{M}^{-\boldsymbol{}}$ is present in the spectrum, the formation of a $\mathrm{M}^{--}$species makes the rationalization of others fragments possible. The ion signal at $\mathrm{m} / z 744.5543$ (A) corresponds to deprotonated $N$-dimethyl-phosphatidylethanolamine species (DMPE) arising from the loss of a methyl radical after electron capture by the positively charged quaternary ammonium ( Scheme S-1). DMPE identity is confirmed by the presence of the ion peak at $m / z 826.5578$ corresponding to a sodium acetate adduct of DMPE. Intramolecular methyl transfer is also observed. The methyl radical, released previously by $\mathrm{M}^{--}$can react with the phosphate group and can then induce the fragmentation of the polar head group, with concomitant elimination of neutral ethylene leading to the formation of a $O$-methyl-phosphatidic acid-like ion detected at $\mathrm{m} / \mathrm{z} 687.4967$ (C). Another homolytic bond cleavage from $\mathrm{M}^{--}$is responsible for the formation of the ion signal (B) at $m / z 699.4964$ due to the loss of the trimethylamine moiety. The elemental composition of the (B) ion is consistent with the detection of its respective acetic acid and sodium acetate adducts at $\mathrm{m} / \mathrm{z}$ 759.5173 and 781.4996 .

Ion peaks labeled (D) and (E) arise from the nucleophilic attack of the phosphate to the carbons at $s n-1$ and $s n-2$ positions, respectively, leading to the fatty acid neutral losses [47]. In the end, the APPI mass spectrum is dominated by carboxylate anion peaks readily allowing the determination of the acyl chain lengths. For example, the ion signals at $\mathrm{m} / \mathrm{z}$ 255.2334 and 281.2484 correspond to palmitate and oleate anions, respectively. (D) and (E) species are not only detected for PC but also for all the other glycerophospholipid classes. The formation of carboxylates and ion (E) is also observed for their 'lyso-' analogues. These fragmentations, together with the carboxylate anion releases, can be useful for locating the acyl chains on the glycerol backbone. Formation of the fivemembered ring species (E) is sterically more favorable than the formation of the six-membered ring (D), leading to a more intense ion peak at $\mathrm{m} / \mathrm{z} 391.2255$. This is confirmed by the higher intensity of the oleate anion peak detected at $\mathrm{m} / \mathrm{z}$ 281.2484 than the palmitate anion peak at $m / z 255.2334$. These intensity ratios on the product-ion spectrum of DMPE generated by ESI have been used in that purpose [54], but collision-induced dissociation can depend on the experimental conditions, such as gas pressure or collision energy. We believe that APPI-induced fragmentations allow similar but more robust determination of acyl chain position on the glycerol backbone.

\section{Ceramides and Cerebrosides}

These sphingolipids exhibit simple APPI mass spectra since only three intense ion signals are detected. The base peak 
corresponds to the deprotonated lipid, followed by acetate and chloride adduct ion peaks. This attachment of chloride observed under APPI conditions has previously been explained by a possible photo-dissociation of chloroform [27]. However, since our normal phase chromatographic system does not use chloroform, the chloride adduct formation is probably due to presence of some chloride anions in the HPLC solvents. Actually, chloride concentrations of 2 and $400 \mathrm{mg} . \mathrm{L}^{-1}$ have been reported in analytical grade acetone and ethyl acetate, respectively, which is high enough to allow an adduct formation [56]. While extensive in-source fragmentation has been highlighted above for most lipid classes, it must be noted that no fragment ion has been detected with ceramide-negative ion analysis. A weak ion signal corresponding to the loss of the galactose moiety has been observed for cerebrosides. Finally, it has to be mentioned that phosphatidylethanolamines, ceramides, and cerebrosides are the only lipid classes that can be analyzed with a comparable sensitivity in both positive and negative ion modes [57].

\section{Sulfatides}

Sulfatides are acidic sphingolipids. Contrary to the positive ion APPI mode which only provided fragment ions, intact $[\mathrm{M}-\mathrm{H}]^{-}$or $[\mathrm{M}-\mathrm{H}+\mathrm{AcONa}]^{-}$sulfatide ions are detected in the negative ion mode (Figure 5). Nevertheless, extensive fragmentation occurs, leading to the straightforward identification of the fatty acyl chain since the ion peak at $\mathrm{m} / \mathrm{z}$ 365.2 can be attributed to nervonate $(\mathrm{C} 24: 1)$. The ceramide skeleton is identified by a series of (A) ions at $\mathrm{m} / \mathrm{z} 628.5$, 646.5 , and 706.5 corresponding to dehydrated ceramide (d18:1/24:1), deprotonated species, and acetic acid adduct, respectively. The membership of this analyte to the

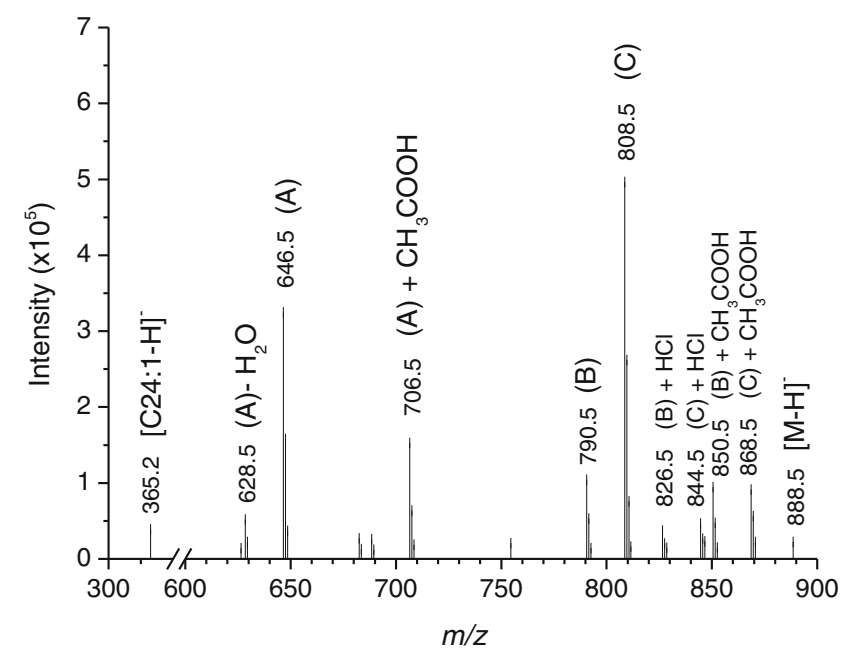

Figure 5. Negative-ion mode APPI mass spectrum of sulfatide (d18:1/24:1). Ceramide-related (A) and GalCerrelated $(C)$ ion series are the major in-source fragment ions observed in negative APPI-MS of sulfatides. Chloride and sodium acetate adducts are also detected glycosphingolipid family is proven by two series of three ions each. The ion peaks at $\mathrm{m} / \mathrm{z} 790.5$ (B) and $\mathrm{m} / \mathrm{z} 808.5$ (C) are assigned to dehydrated and deprotonated GalCer. Their respective chloride adducts are detected at $\mathrm{m} / \mathrm{z} 826.5$ and 844.5 , confirming the affinity of chloride to sphingolipids. Finally, the GalCer ion series is completed by the presence of acetic acid adducts at $m / \mathrm{z} 850.5$ and 868.5 , respectively.

Under APPI conditions, the main observed fragmentations are the neutral loss of sulfuric anhydride $(-80 \mathrm{Da})$ and of galactosylpyranose-3-sulfate (-242 Da). ESI-MS/MS analysis of sulfatides performed with a triple quadrupole lead to a major ion peak at $m / z 97$ corresponding to a sulfate anion whereas an ion-trap $\mathrm{MS}^{2}$ spectrum presents intense ion signal at $\mathrm{m} / \mathrm{z} 241$, arising from the sulfated sugar [58]. No GalCer-related ions have been reported in these ESI-MS/ MS experiments.

A carboxylate signal is detected in the APPI spectrum with a better signal-to-noise ratio than with tandem MS. The chain length can thus be easily determined by APPI-MS. A more detailed structural characterization can be obtained by lowenergy CID with triple quadrupole or ion trap analyzers since charge-remote fragmentations have been observed in tandem mass spectra. However, a detailed characterization of double bond position may not be always necessary for lipid profiling, and APPI can thus provide a sufficient level of knowledge, intermediate between full scan and product ion spectra in ESI.

\section{Sphingomyelin}

We demonstrated in the positive-ion mode that sphingomyelin has both a sphingolipid-like and a PC-like behavior. The same trend is observed in the negative-ion APPI mass spectrum which present a wealth of structural information since no less than 18 ion peaks have been annotated (Figure 6). The presence of both a quaternary ammonium

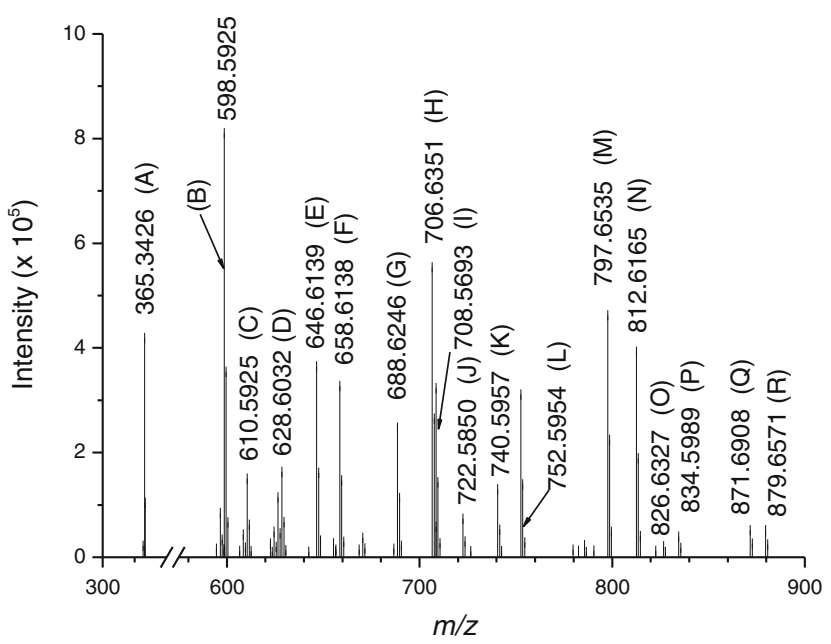

Figure 6. Negative-ion mode APPI mass spectrum of sphingomyelin (d18:1/24:1). This spectrum combines both sphingolipid-like and PC-like fragmentation behaviors and exhibits numerous adducts 
in the SM structure and of acetic acid in the mobile phase is responsible, as for $\mathrm{PC}$, for an ambiguity for the assignment of the ion peak at $m / z$ 812.6, which can be either a $\mathrm{M}^{-}$or an acetic acid adduct of the fragment obtained after loss of trimethylamine. The SM family has thus been investigated with the LTQ-Orbitrap and all relevant ion peaks have been annotated with a sub-2 ppm mass error.

Ion signals can be divided in four groups: ion (A) at $\mathrm{m} / \mathrm{z}$ 365.3426 allows identifying the long fatty acyl chain as nervonic acid (C24:1). Ions $(\mathrm{B})$ to $(\mathrm{H})$ are fragments related to ceramide moiety, whereas ions (I) to (M) retain the phosphate group. Finally, ions $(\mathrm{N})$ to $(\mathrm{R})$ are acetic acid adducts of the latter fragments.

An acetate adduct of intact sphingomyelin is detected at $\mathrm{m} / \mathrm{z} 871.6908$ (Q). The (M) labeled ion is generated from $\mathrm{M}^{--}$by $\mathrm{N}$-demethylation, according to the same mechanism as for PC, the (R) labeled ion is its sodium acetate adduct, and the $(\mathrm{L})$ ion is obtained by a loss of the trimethylamine group from $\mathrm{M}^{-}$. The spectrum also shows the acetic acid $(\mathrm{N})$ and sodium acetate (P) adduct ion peaks of (L). An intramolecular methyl transfer occurs, leading to the formation of the deprotonated $O$-methyl-ceramide-1-phosphate $(\mathrm{K})$ and to a minor dehydrated ion $(\mathrm{J})$. The formation of the ion labeled $(\mathrm{O})$ is supposed to combine both a methyl transfer and a trimethylamine loss. The resulting fully substituted phosphate is observed as an acetate adduct ion. Finally, the ceramide-1-phosphate moiety is also detected as a dehydrated ion (I).

Further fragmentations are characterized by the complete loss of the phosphocholine head-group. The ceramide moiety is detected as deprotonated (E), dehydrated (D), and di-dehydrated ion species $(\mathrm{C})$. Acetate adducts $(\mathrm{G})$ and $(\mathrm{H})$ correspond, respectively, to ions (D) and (E). An intense ion peak (B) and its acetic acid adduct (F) arise from consecutive losses of formaldehyde and of water as observed by ESI-MS/MS [59].

The negative-ion APPI-MS provides an interesting tool for the structural analysis of sphingomyelin since both the phosphocholine head-group and ceramide skeleton can be fully characterized. However, the distribution of the ion beam in so many fragment ions is responsible for a 20 -fold decrease in signal-to-noise ratio compared to the positive-ion mode.

\section{Application to Lipidomic Studies}

Atmospheric pressure photoionization is particularly suited to the hyphenation of normal-phase liquid chromatography with mass spectrometry. This ionization source uses the favorable physico-chemical properties of NPLC solventslow ionization potential, high volatility - leading to sensitive analysis of apolar and medium polar lipids. As extensively described, APPI induces in-source fragmentations that is greatly useful to characterize lipid structures.

Lipidomic studies are aimed to highlight discriminating lipid signals between groups of samples, differing for instance by their treatment or health status and then to elucidate the structure of these molecules, either by interpreting their fragmentation spectra or by comparing them to an authentic standard.

APPI-induced fragmentations generate signal redundancy that can be a problem in the frame of complex biological sample analysis since it can slow down chemometric treatment and spectra interpretation and finally the whole complex process of structural elucidation. However, it has to be noticed that is not a specific feature of APPI since fragment ions due to in-source CID, adducts with alkaline metals, and dimeric ions are also detected in ESI.

The coelution in normal-phase liquid chromatography of lipids from a single class exhibiting the same fragmentation patterns will make spectra interpretation easier. Ion peaks representative of lipid species will be clustered as described and separated by combinations of 2 or $28 \mathrm{Da}$ increments, corresponding to double bond and two methylene mass shifts, respectively. The same principles apply to all acyl chain-containing fragment ions.

Besides, mathematical tools are available to reduce signal redundancy. The use of auto-correlation matrices, originally designed for NMR data processing [60], has proved its efficiency in mass spectrometry-based metabolomics to relate fragment ions and adducts to the protonated or deprotonated molecule [61].

Consequently, the use of a combination of adequate liquid chromatography system and data processing method could allow analyzing complex biological samples while taking advantage of APPI in-source fragmentation, in order to collect both lipid fingerprint and structural information in the same experiment, thus partly avoiding the need of reinjection for MS/MS analysis that is particularly useful for volume-limited samples. More generally, a speed-up in the analytical workflow can thus be expected from the use of APPI for lipidomic studies.

\section{Conclusion}

In the frame of coupling normal-phase liquid chromatography to mass spectrometry for lipid profiling applications, the use of atmospheric pressure photoionization has proven its versatility. Indeed, acidic species have been detected in the positive ion mode, whereas quaternary ammonium-containing species have been successfully investigated in negative-ion mode. Moreover apolar lipids have been analyzed with an enhanced sensitivity, compared to ESI. Finally, APPI provides fast but detailed structural information thanks to in-source fragmentations, which are either similar to those obtained by CID or specific to APPI. In the latter case, they often involve radical dissociations. A wide range of precursor ions, arising from different parts of the lipid structure, is thus available for further CID experiments, allowing pseudo-MS ${ }^{3}$ to be performed, enhancing the capabilities of triple stage quadrupole mass spectrometers. 


\section{Acknowledgments}

M.G. acknowledges Technologie Servier for funding. The authors thank the Federative Research Institute IFR 141 for financing this study through the Young Scientist Grant.

\section{References}

1. Fahy, E., Subramaniam, S., Brown, H.A., Glass, C.K., Merrill Jr., A.H. Murphy, R.C., Raetz, C.R., Russell, D.W., Seyama, Y., Shaw, W., Shimizu, T., Spener, F., van Meer, G., VanNieuwenhze, M.S., White, S. H., Witztum, J.L., Dennis, E.A.: A comprehensive classification for lipids. J. Lipid Res. 46, 839-861 (2005)

2. Fletcher, A.: The cell membrane and receptors. Anaesth. Intensive Care Med. 11, 384-388 (2010)

3. Lingwood, D., Simons, K.: Lipid rafts as a membrane-organizing principle. Science 327, 46-50 (2010)

4. Beller, M., Thiel, K., Thul, P.J., Jäckle, H.: Lipid droplets: A dynamic organelle moves into focus. FEBS Lett. 584, 2176-2182 (2010)

5. Farooqui, A.A., Ong, W., Farooqui, T.: Lipid mediators in the nucleus: Their potential contribution to Alzheimer's disease. Biochim. Biophys. Acta 1801, 906-916 (2010)

6. White, T., Bursten, S., Federighi, D., Lewis, R.A., Nudelman, E.: High resolution separation and quantification of neutral lipid and phospholipid species in mammalian cells and sera by multi-one-dimensional thin-layer chromatography. Anal. Biochem. 258, 109-117 (1998)

7. Gutnikov, G.: Fatty acid profiles of lipid samples. J. Chromatogr. B Biomed. Appl. 71, 71-89 (1995)

8. Deschamps, F.S., Chaminade, P., Ferrier, D., Baillet, A.: Assessment of the retention properties of poly(vinyl alcohol) stationary phase for lipid class profiling in liquid chromatography. J. Chromatogr. A 928, 127137 (2001)

9. Vernooij, E., Brouwers, J., Kettenes-van den Bosch, J., Crommelin, D.: RPHPLC/ESIMS determination of acyl chain positions in phospholipids. J. Sep. Sci. 25, 285-289 (2002)

10. Pulfer, M., Murphy, R.C.: Electrospray Mass Spectrometry of Phospholipids. Mass Spectrom. Rev. 22, 332-364 (2003)

11. Lu, B., Zhang, Y., Wu, X., Shi, J.: Separation and determination of diversiform phytosterols in food materials using supercritical carbon dioxide extraction and ultraperformance liquid chromatographyatmospheric pressure chemical ionization-mass spectrometry. Anal. Chim. Acta 588, 50-63 (2007)

12. Byrdwell, W.C., Neff, W.E.: Dual parallel electrospray ionization and atmospheric pressure chemical ionization mass spectrometry (MS), MS/ MS and MS/MS/MS for the analysis of triacylglycerols and triacylglycerol oxidation products. Rapid Commun. Mass Spectrom. 16, 300-319 (2002)

13. Hsu, F.F., Turk, J.: Structural characterization of triacylglycerols as lithiated adducts by electrospray ionization mass spectrometry using low-energy collisionally activated dissociation on a triple stage quadrupole instrument. J. Am. Soc. Mass Spectrom. 10, 587-599 (1999)

14. Levêque, N.L., Héron, S., Tchapla, A.: Regioisomer characterization of triacylglycerols by non-aqueous reversed-phase liquid chromatography/ electrospray ionization mass spectrometry using silver nitrate as a postcolumn reagent. J. Mass Spectrom. 45, 284-296 (2010)

15. Robb, D.B., Covey, T.R., Bruins, A.P.: Atmospheric pressure photoionisation: an ionization method for liquid chromatography-mass spectrometry. Anal. Chem. 72, 3653-3659 (2000)

16. Zhou, S.N., Reiner, E.J., Marvin, C., Kolic, T., Riddell, N., Helm, P., Dorman, F., Misselwitz, M., Brindle, I.D.: Liquid chromatographyatmospheric pressure photoionization tandem mass spectrometry for analysis of 36 halogenated flame retardants in fish. J. Chromatogr. A 1217, 633-641 (2010)

17. Smith, D.R., Robb, D.B., Blades, M.W.: Comparison of dopants for charge exchange ionization of nonpolar polycyclic aromatic hydrocarbons with reversed-phase LC-APPI-MS. J. Am. Soc. Mass Spectrom. 20, 73-79 (2009)

18. Zhang, F., Bartels, M.J., Geter, D.R., Carr, M.S., McClymount, L.E., Marino, T.A., Klecka, G.M.: Simultaneous quantitation of testosterone, estradiol, ethinyl estradiol, and 11-ketotestosterone in fathead minnow fish plasma by liquid chromatography/positive atmospheric pressure photoionization tandem mass spectrometry. Rapid Commun. Mass Spectrom. 24, 958-964 (2010)

19. Debois, D., Giuliani, A., Laprévote, O.: Fragmentation induced in atmospheric pressure photoionization of peptides. J. Mass Spectrom. 41, 1554-1560 (2006)

20. Delobel, A., Halgand, F., Laffranchise-Gosse, B., Snijders, H., Laprévote, O.: Characterization of hydrophobic peptides by atmospheric pressure photoionization-mass spectrometry and tandem mass spectrometry. Anal. Chem. 75, 5961-5968 (2003)

21. Giuliani, A., Debois, D., Laprévote, O.: Study of a bisquaternary ammonium salt by atmospheric pressure photoionization mass spectrometry. Eur. J. Mass Spectrom. 12, 189-197 (2006)

22. Bagag, A., Laprévote, O., Hirsch, J., Kováčik, V.: Atmospheric pressure photoionization mass spectrometry of per-O-methylated oligosaccharides related to d-xylans. Carbohydr. Res. 343, 2813-2818 (2008)

23. Bagag, A., Giuliani, A., Laprévote, O.: Atmospheric pressure photoionization of oligodeoxyribonucleotides. Eur. J. Mass Spectrom. 14, 71-80 (2008)

24. Wang, G., Hsieh, Y., Korfmacher, W.A.: Comparison of Atmospheric Pressure Chemical Ionization, Electrospray Ionization, and Atmospheric Pressure Photoionization for the Determination of Cyclosporin A in Rat Plasma. Anal. Chem. 77, 541-548 (2005)

25. Cai, S.S., Syage, J.A.: Comparison of atmospheric pressure photoionization, atmospheric pressure chemical ionization and electrospray ionization mass spectrometry for the analysis of lipids. Anal. Chem. 78, 1191-1199 (2006)

26. Karuna, R., von Eckardstein, A., Rentsch, K.M.: Dopant assistedatmospheric pressure photoionization (DA-APPI) liquid chromatography-mass spectrometry for the quantification of 27-hydroxycholesterol in plasma. J. Chromatogr. B 877, 261-268 (2009)

27. Delobel, A., Roy, S., Touboul, D., Gaudin, K., Germain, D.P., Baillet, A., Brion, F., Prognon, P., Chaminade, P., Laprévote, O.: Atmospheric pressure photoionization coupled to porous graphitic carbon liquid chromatography for the analysis of globotriaosylceramides. Application to Fabry disease. J. Mass Spectrom. 41, 50-58 (2006)

28. Roy, S., Delobel, A., Gaudin, K., Touboul, D., Germain, D.P., Baillet, A., Prognon, P., Laprévote, O., Chaminade, P.: Liquid chromatography on porous graphitic carbon with atmospheric pressure photoionization mass spectrometry and tandem mass spectrometry for the analysis of glycosphingolipids. J. Chromatogr. A 1117(2), 154-62 (2006)

29. Delobel, A., Touboul, D., Laprévote, O.: Structural characterization of phosphatidylcholines by atmospheric pressure photoionization mass spectrometry. Eur. J. Mass Spectrom. 11, 409-417 (2005)

30. Graeve, M., Janssen, D.J.: Improved separation and quantification of neutral and polar lipid classes by HPLC-ELSD using a monolithic silica phase: application to exceptional marine lipids. J. Chromatogr. B Anal. Technol. Biomed. Life Sci. 877, 1815-1819 (2009)

31. Higgins, D., Carlson, J., Van Nest, G.: MF59 adjuvant enhances the immunogenicity of influenza vaccine in both young and old mice. Vaccine 14, 478-484 (1996)

32. Chen, A., Poulter, C.D.: Purification and characterization of farnesyl diphosphate/geranylgeranyl diphosphate synthase. A thermostable bifunctional enzyme from Methanobacterium thermoautotrophicum. J. Biol. Chem. 268(15), 11002-11007 (1993)

33. Acimovic, J., Lövgren-Sandblom, A., Monostory, K., Rozman, D., Golicnik, M., Lutjohann, D., Björkhem, I.: Combined gas chromatographic/mass spectrometric analysis of cholesterol precursors and plant sterols in cultured cells. J. Chromatogr. B 877, 2081$2086(2009)$

34. Grigoriadou, D., Androulaki, A., Psomiadou, E., Psimidou, M.Z.: Solid phase extraction in the analysis of squalene and tocopherols in olive oil. Food Chem. 105, 675-680 (2007)

35. Syage, J.A.: Mechanism of $[\mathrm{M}+\mathrm{H}]^{+}$formation in photoionization mass spectrometry. J. Am. Soc. Mass Spectrom. 15, 1521-1533 (2004)

36. NIST Chemistry WebBook.

37. Yuan, T., Zhang, L., Zhou, Z., Xie, M., Ye, L., Qi, F.: Pyrolysis of $n$ heptane: Experimental and theoretical study. J. Phys. Chem. A 115, 1593-1601 (2011)

38. Cai, S.S., Short, L.C., Syage, J.A., Potvin, M., Curtis, J.M.: Liquid chromatography-atmospheric pressure photoionization-mass spectrometry analysis of triacylglycerol lipids - effects of mobile phases on sensitivity. J. Chromatogr. A 1173, 88-97 (2007)

39. Carpenter, K.L., Taylor, S.E., Ballantine, J.A., Fussell, B., Halliwell, B., Mitchinson, M.J.: Lipids and oxidized lipids in human atheroma and normal aorta. Biochim. Biophys. Acta 1167, 121-30 (1993) 
40. Panchal, M., Loeper, J., Cossec, J.-C., Perruchini, C., Lazar, A., Pompon, D., Duyckaerts, C.: Enrichment of cholesterol in microdissected Alzheimer's disease senile plaques as assessed by mass spectrometry. J. Lipid Res. 51, 598-605 (2010)

41. Varga, M., Bartók, T., Mesterházy, A.: Determination of ergosterol in Fusarium-infected wheat by liquid chromatography-atmospheric pressure photoionization mass spectrometry. J. Chromatogr. A 1103, 278283 (2006)

42. Hsu, F.-F., Turk, J.: Electrospray Ionization/Tandem Quadrupole Mass Spectrometric Studies on Phosphatidylcholines: The Fragmentation Processes. J. Am. Soc. Mass Spectrom. 14, 352-363 (2003)

43. Hsu, F.-F., Turk, J., Thukkani, A.K., Messner, M.C., Wildsmith, K.R., Ford, D.A.: Characterization of alkylacyl, alk-1-enylacyl, and lyso subclasses of glycerophosphocholine by tandem quadrupole mass spectrometry with electrospray ionization. J. Mass Spectrom. 38, 752-763 (2003)

44. Hsu, F.-F., Turk, J., Williams, T.D., Welti, R.: Electrospray ionization multiple stage quadrupole ion-trap and tandem quadrupole mass spectrometric studies on phosphatidylglycerol from Arabidopsis leaves. J. Am. Soc. Mass Spectrom. 18, 783-790 (2007)

45. Hsu, F.-F., Turk, J.: Characterization of phosphatidylinositol, phosphatidylinositol-4-phosphate, and phosphatidylinositol-4,5-bisphosphate by electrospray ionization tandem mass spectrometry: a mechanistic study. J. Am. Soc. Mass Spectrom 11, 986-999 (2000)

46. Hsu, F.-F., Turk, J.: Charge-driven fragmentation processes in diacyl glycerophosphatidic acids upon low-energy collisional activation. A mechanistic proposal. J. Am. Soc. Mass Spectrom. 11, 797-803 (2000)

47. Hsu, F.-F., Turk, J.: Electrospray ionization with low-energy collisionally activated dissociation tandem mass spectrometry of glycerophospholipids: Mechanisms of fragmentation and structural characterization. J. Chromatogr. B 877, 2673-2695 (2009)

48. Muñoz-Garcia, A., Ro, J., Brown, J.C., Williams, J.B.: Identification of complex mixtures of sphingolipids in the stratum corneum by reversedphase high-performance liquid chromatography and atmospheric pressure photospray ionization mass spectrometry. J. Chromatogr. A 1133, 58-68 (2006)

49. Kim, H.Y., Yergey, J.A., Salem Jr., N.: Determination of eicosanoids, phospholipids and related compounds by thermospray liquid chromatography-mass spectrometry. J. Chromatogr. 394, 155-170 (1987)

50. Hsu, F.-F., Turk, J., Stewart, M.E., Downing, D.T.: Structural studies on ceramides as lithiated adducts by low energy collisional-activated dissociation tandem mass spectrometry with Electrospray ionization. $J$. Am. Soc. Mass Spectrom. 13, 680-695 (2002)

51. Suzuki, K., Kameyama, A., Tachibana, K., Narimatsu, H., Fukui, K.: Computationally and Experimentally Derived General Rules for
Fragmentation of Various Glycosyl Bonds in Sodium Adduct Oligosaccharides. Anal. Chem. 81, 1108-1120 (2009)

52. Hsu, F.-F., Turk, J.: Structural determination of glycosphingolipids as lithiated adducts by Electrospray ionization mass spectrometry using low-energy collisional-activated dissociation on a triple stage quadrupole instrument. J. Am. Soc. Mass Spectrom. 12, 61-79 (2001)

53. Han, X., Holtzman, D.M., McKneel Jr., D.W., Kelly, J., Morris, J.C.: Substantial sulfatide deficiency and ceramide elevation in very early Alzheimer's disease: potential role in disease pathogenesis. J. Neurochem. 82, 809-818 (2002)

54. Hsu, F.-F., Turk, J.: Structural determination of sphingomyelin by tandem mass spectrometry with Electrospray ionization. J. Am. Soc. Mass Spectrom. 11, 437-449 (2000)

55. Kauppila, T.J., Kotiaho, T., Kostiainen, R., Bruins, A.P.: Negative ion Atmospheric Pressure Photoionization-Mass Spectrometry. J. Am. Soc. Mass Spectrom. 15, 203-211 (2004)

56. Sergeev, G.M., Blinova, M.S.: Ion chromatography of impurities in organic solvents. J. Chromatogr. A 847, 345-349 (1999)

57. Gaudin, M.; Imbert, L.; Libong, D.; Chaminade, P.; Loiseau, P. M.; Allegrand, Auzeil, N.; Touboul, D.; Laprévote, O. Lipid analysis of biological samples by normal-phase liquid chromatography / atmospheric pressure photoionization mass spectrometry. Proceedings of the 58th ASMS Conference on Mass Spectrometry and Allied Topics, Salt Lake City, Utah, May 23-27, 2010. (1945)

58. Hsu, F.-F., Turk, J.: Studies on Sulfatides by Quadrupole Ion-Trap Mass Spectrometry with Electrospray Ionization: Structural Characterization and the Fragmentation Processes that Include an Unusual Internal Galactose Residue Loss and the Classical Charge-Remote Fragmentation. J. Am. Soc. Mass Spectrom. 15, 536-546 (2004)

59. Han, X.: Characterization and Direct Quantitation of Ceramide Molecular Species from Lipid Extracts of Biological Samples by Electrospray Ionization Tandem Mass Spectrometry. Anal. Biochem. 302, 199-212 (2002)

60. Cloarec, O., Dumas, M.-E., Craig, A., Barton, R.H., Trygg, J., Hudson, J., Blancher, C., Gauguier, D., Lindon, J.C., Holmes, E., Nicholson, J.: Statistical Total Correlation Spectroscopy: An Exploratory Approach for Latent Biomarker Identification from Metabolic ${ }^{1} \mathrm{H}$ NMR Data Sets. Anal. Chem. 77, 1282-1289 (2005)

61. Werner, E., Croixmarie, V., Umbdenstock, T., Ezan, E., Chaminade, P., Tabet, J.-C., Junot, C.: Mass Spectrometry-Based Metabolomics: Accelerating the Characterization of Discriminating Signals by Combining Statistical Correlations and Ultrahigh Resolution. Anal. Chem. 80, 4918-4932 (2008) 